\title{
Two-Stage Optimization Model for Smart House Daily Scheduling Considering User Perceived Benefits
}

\author{
Changhong Deng, Sijie Zhang, Wei Yang, Weiwei Yao, Jin Tan and Zhengyi Liu \\ School of Electrical Engineering, Wuhan University, Wuhan, Hubei Province, China
}

\begin{abstract}
Smart house scheduling is the grid terminal demand response based on the reference signal, the electricity market price. In this paper, the user consumption perceived benefits and the system running cost were considered. In the intelligent house system with energy storage device, the load control model and the energy storage control model were established, and the two-stage optimization scheduling was carried out for the smart house appliances to get rid of the disturbance of load uncertainty. The first stage took the flexible load as the control object, and the genetic algorithm was proposed to provide a schedule for smart home appliances. The second stage considered the energy storage device as the control object and a particle swarm algorithm was used to generate a charge/discharge rates schedule for the battery. The optimal solution of the first stage optimization control participated in the second stage optimization control in the form of the load curve. The fitness value of the optimal solution of the load control stage was taken as the minimum objective function constraint of the energy storage control stage, thus further reducing the electricity cost of the terminal-user. The simulation example in MATLAB verifies the effectiveness of models.
\end{abstract}

Keywords-two-stage optimization model; smart house daily scheduling; consumption perceived benefits

\section{INTRODUCTION}

Demand-side response, behavior game between power suppliers and power distributors, household micro-grids, and electric car are the main directions of current research on power market. Demand Side Response includes three types of applications in the retail market [1]: interruptible load projects, electricity repurchase programs, dynamic tariff projects. The research on home micro-grid mainly focuses on the demand side such as energy storage control, new energy utilization and load matching. From the viewpoint of load peak regulation, paper[2] proposed the optimization strategy of equivalent virtual energy storage for temperature control system and participating in the peak regulation of regional distribution network based on the physical characteristics of air conditioning-building. From the perspective of game theory in the electricity market, Ref. [3] established the distribution multi-participation market model and the electricity market model considering the behavior of the power generation company. Based on the characteristics of the incomplete game, co-evolutionary algorithm was applied to solve the BayesianNash equilibrium problem in the market game. From the perspective of micro-grid, literature [4] established a joint scheduling model of photovoltaic-storage based on micro-grid to optimize the distribution ratio of residual power. By reasonably allocating the residual power of photovoltaic power generation and energy storage in each period, Ref. [5] proposed different scheduling strategies. From the perspective of demand-side multi-user energy coordination, the multi-user energy dispatching method is issued in paper[6], with the goal of reducing electricity consumption costs in the region. From the perspective of user experience, Ref.[7] designed smart home energy management system to save energy costs by delaying or advancing the flexible load working period to match the power curve of the house micro-power supply.

Considering the characteristics of energy storage and house appliances, this paper proposes a two-phase coordinated operation strategy to meet the demand of power consumption and user satisfaction, which called Grid terminal intelligent power consumption technology. In this paper, the energy storage scheduling method is divided into two stages. Firstly, the flexible load is optimally arranged to minimize the operating cost. Then, based on the first-stage load curve, energy storage system is economically dispatched in the intelligent home. Finally, the economic and rationality of the method is verified by simulation.

\section{The First Stage Optimization MODEL WITH FLEXIBLE LOAD AS THE CONTROL OBJECT}

\section{A. Optimal Scheduling Model}

\section{1) Load classification}

This article classifies the load by the time or power elasticity of the house appliances. The time elasticity of load is the shift cost when the electricity appliances transfer from one period to another. The power elasticity of load is the added cost when the load electricity consumption changes[8]. The lower the cost, the greater the corresponding characteristic elasticity.

TABLE I. LOAD CLASSIFICATION IN SMART HOUSE

\begin{tabular}{cccc}
\hline \multicolumn{2}{c}{ Appliance Type } & Time & Power \\
\hline Uncontrollable & & $\times$ & $\times$ \\
\hline \multirow{2}{*}{ Controllable } & T-type & $\sqrt{ }$ & $\times$ \\
\cline { 2 - 4 } & P-type & $\times$ & $\sqrt{ }$
\end{tabular}

The load elasticity is infinity which means the application is uncontrollable such as rice cookers, whose working hours and power consumption are not adjustable because once these parameters change will seriously affect the user's experience [9], that is also called, rigid load. Flexible load, with load 
flexibility, is controllable in operation time(T-type) or controllable in operational power(P-type).

\section{2) Load volatility set}

According to the rated power of each flexible load and the user's habit in electricity consumption, it is possible to obtain boundary reference for each uncertain parameter. For P-type appliances, $\tilde{P}_{k}$ - the base value of power; $\varepsilon_{k}$ - the disturbance quantity of power; For T-type appliances, $\left[\alpha_{k}, \beta_{k}\right]$ is the working period; $t_{k}^{\text {start }}$ - start time, $t_{k}^{\text {end }}$ - ending time; $d_{k}$ - the total running time are also available. The above parameters are expressed in the following indefinite set,

$$
\begin{gathered}
U_{1}=\left\{P_{k}(t)=\tilde{P}_{k}(t)+\varepsilon_{k}(t) ; \underline{\varepsilon_{k}} \leq \varepsilon_{k}(t) \leq \overline{\varepsilon_{k}}\right\} \\
U_{2}=\left\{\sum_{t=1}^{T} x_{k}^{t}=d_{k} ; d_{k} \leq \beta_{k}-\alpha_{k} ; \alpha_{k} \leq t_{k}^{\text {start }} \leq t_{k}^{\text {end }} \leq \beta_{k}\right\}
\end{gathered}
$$

where $x_{k}^{t}$ indicates the operating status of the appliance.

\section{3) Electricity consumption perceived benefits model}

Electricity consumption perceived benefits, also called consumption satisfaction, is the satisfaction or comfort obtained by users through electricity consumption, which is mainly related to electricity price and electricity amount.

The relationship between the three parameters can be summarized as following:

- electricity consumption amount is proportional to the consumer's comfort degree

- user's satisfaction increased when electricity price rises, but the increase trend will gradually slowdown

The above characteristics can be expressed in terms of the logarithmic utility function that guides the resource allocation in the microeconomics.

$$
f(\alpha, x)=\alpha \operatorname{In}\left(x^{k}+1\right), \forall \alpha>0, x \geq 0, k \geq 1
$$

Logarithmic utility function has two features,

- The function value increases as $x$ or $\alpha$ increases, and the function minimum:

$$
f(\alpha, x)_{\min }=\left.\alpha \operatorname{In}\left(x^{k}+1\right)\right|_{x=0}=0
$$

- The second derivative of the function $\frac{\partial^{2} f}{\partial x^{2}}<0$, which means monotonically increasing trend of the function value gradually weakened as $X$ increases .

Considering the logarithmic utility function has same properties, the relationship between the grid electricity price, electricity consumption and the user satisfaction degree in this paper is described as follows,

$$
e f f_{k}=c_{k} \operatorname{In}\left(p_{k}+1\right)
$$

where $C_{k}$ is the consumption factor at time slot $k$, which used to represent the user satisfaction degree obtained by the power consumption, and its value is equal to the electricity price; $p_{k}$ is the consumer's power consumption amount at time slot $k$.

\section{B. Objection of the First Optimization Model}

The first stage of smart home scheduling use the positive benefits of electricity as the goal, the equation is written as:

$$
F_{1}=\max \left(f_{1}-f_{2}\right)
$$

where $f_{1}$ is consumption satisfaction; $f_{2}$, the daily cost of electricity.

The user's power consumption satisfaction and cost of electricity are represented respectively in equations (7)(8):

$$
\begin{gathered}
f_{1}=\sum_{k=1}^{T} e f f_{k} \\
f_{2}=\sum_{k=1}^{T} c_{k} p_{k}
\end{gathered}
$$

where $c_{k}$ is the electricity price; $p_{k}$ is the electricity consumption amount.

\section{ThE SECOND Optimization MODEL WITH ENERGY STORAGE SYSTEM AS CONTROL OBJECT}

\section{A. Energy Storage Scheduling Model}

As a energy storage components, battery has two working modes: charging and discharging. The energy storage efficiency of the battery is greatly affected by the temperature. During the continuous charging and discharging process, the internal temperature of the battery rises, which affects the internal chemical reaction in the battery. When the ambient temperature varies between 10 and $30{ }^{\circ} \mathrm{C}$, the internal chemical reaction of the battery is favorable and the storage efficiency is high. So the battery pack is generally placed indoors and the ambient temperature is maintained at 25 degrees Celsius [10-13].

The battery model is formulated in equation EQ-9:

$$
S O C=\frac{\sum_{i=1}^{N} p_{\text {exchange }} \Delta t}{E_{\max }}+\frac{E_{\text {initial }}}{E_{\max }}
$$


Battery residual energy is characterized in equation EQ-10:

$$
E_{b, t}=\operatorname{SOC}_{t} C
$$

where $S O C_{t}$ is the state of charge; $E_{b, t}$, the remaining stored energy; $C$, the rated capacity of the battery.

\section{B. Objection of the Second Optimization Model}

Considering the power supply characteristics of energy storage system, the operating cost of smart home will be composed of two parts: electricity purchase costs and electricity sales revenue. When the storage discharge power is higher than the actual load demand, the remaining power will be sold to the grid then charges a certain fee. The optimization goal of the second phase is to minimize the system operating cost in the grid-connected mode. The mathematical expression is as follows:

$$
\begin{gathered}
F_{2}=\min \left(f_{g}-f_{s}\right) \\
f_{g}=\sum_{k=1}^{T} C_{g, k} P_{g, k} \\
f_{s}=\sum_{k=1}^{T} C_{s, k} P_{s, k}
\end{gathered}
$$

where, $f_{g}$ is the purchase electricity costs and $f_{s}$ is the sales revenue; $C_{g, k}$, the purchase electricity price; $C_{s, k}$, the electricity sales price; $P_{g, k}$, the power purchases from the grid; $P_{s, k}$, the power sales to the grid.

\section{Constraints of the Second Model}

1) Minimum objective function constrains

The second optimized system operating costs $F_{2}$ shall not be higher than the first system operating costs $f_{2}$.

$$
F_{2}<f_{2}
$$

\section{2) Battery constraints}

The state of charge is an important physical parameter of the battery. Low state of charge can cause voltage instability, and the floating phenomenon may occur when the state of charge is too high. In order to prolong the battery service life, it is necessary to limit the state of charge.

$$
S O C_{\min } \leq S O C_{t} \leq S O C_{\max }
$$

The discharge power will be limited.

$$
-\frac{0.2 C}{T_{s}} \leq P_{b, t} \leq \frac{0.2 C}{T_{s}}
$$

where $T_{s}$ is duration of each time interval.

The cumulative discharge power is a measure of battery life. The low cumulative discharge is beneficial to prolong the working life of the battery, but it is not conducive to the economic operation of the system. If the cumulative discharge is too high, it is helpful to smart house scheduling but will reduce the service life of the battery. Therefore, constraints of the cumulative battery discharge power are given by the following equations:

$$
\begin{gathered}
E_{d T}=\sum_{t=1}^{T} P_{d . t} T_{s} \\
E_{d T}^{\min } \leq E_{d T} \leq E_{d T}^{\max }
\end{gathered}
$$

where $E_{d T}$ is the accumulative discharge power.

\section{SMART HOME OPTIMIZATION CONTROL ALGORITHM}

The genetic algorithm is applied to control all appliances. The particle swarm optimization algorithm is applied to produce schedule for the energy storage system.

\section{SIMULATION EXAMPLE}

\section{A. Parameter}

TABLE II. TIME CONTROLLABLE LOAD

\begin{tabular}{lccc}
\hline Appliance & $\begin{array}{c}\text { Profile } \\
\text { Length }\end{array}$ & Time Range & $\begin{array}{c}\text { Rated } \\
\text { Power }\end{array}$ \\
\hline Oven & 1 & $24-28$ & $1500 \mathrm{w}$ \\
Clothes Washer & 6 & $1-96$ & $400 \mathrm{w}$ \\
Vacuum Cleaner & 4 & $1-96$ & $600 \mathrm{w}$ \\
Dishwasher & 7 & $67-96$ & $1000 \mathrm{w}$ \\
Dryer & 8 & $17-96$ & $1500 \mathrm{w}$ \\
\hline
\end{tabular}

TABLE III. POWER CONTROLLABLE LOAD

\begin{tabular}{lccc}
\hline Appliance & Profile Length & Rated Power & Scope \\
\hline Refrigerator & 96 & $200 \mathrm{w}$ & $\pm 10 \%$ \\
Air conditioner & 96 & $1200 \mathrm{w}$ & $\pm 10 \%$
\end{tabular}

This paper chooses time-of-use model of electricity price. The electricity price, taking the price curve of the PJM market in the United States as of July 10, 2017 as a reference, is updated every hour, as shown in FIG. 2. 


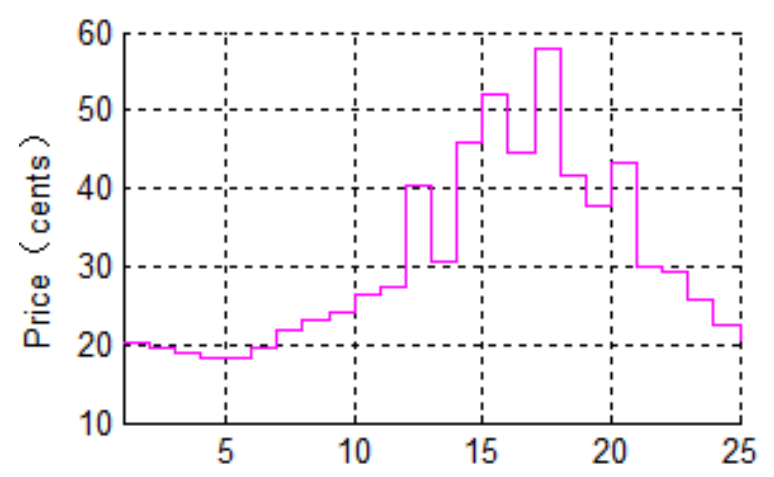

FIGURE I. TIME-OF-USE TARIFF

\section{B. Example Analysis}

1) Analysis of Two Stage Energy Storage Control Results

As shown in Figure 3, the source power distribution can be seen. Before 8 o'clock, the grid supplies power to the smart house. The electricity price is at the lowest level of the whole day. At this time, the storage battery generally charges, therefore, the purchasing power is high during this period. From 8:00 to 9:00, the household electric power reaches the maximum value of $5 \mathrm{KW}$. When the electricity price is in the low period, the purchase cost is also low and the storage level of the battery is high. During 10 o'clock to 12 o'clock, in accordance with the instructions of the optimal scheduling charge, the battery transfers electricity to obtain economic benefits. During 13:00 to 21:00, SOC is high, so the battery discharges to reduce the cost of electricity purchase and ease the peak load. Before 22 o'clock, the battery discharges to reduce the amount of electricity purchased by the user when the electricity price is high. At 23:00 to 1:00, the battery charges to maintain the initial SOC for the next cycle.

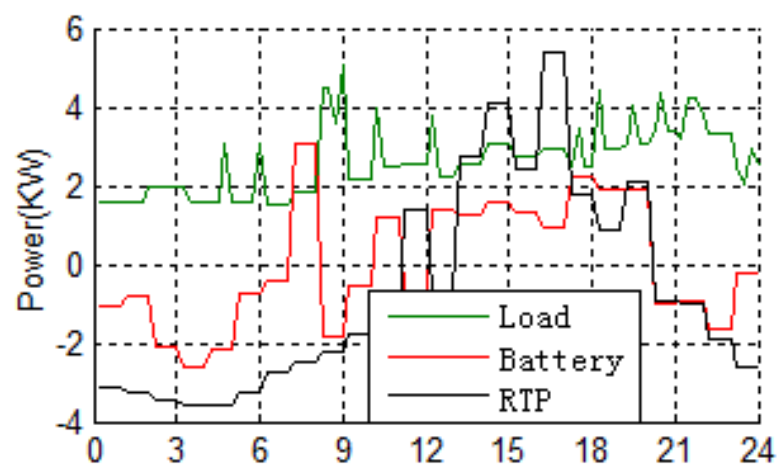

FIGURE II. DAILY OPTIMAL OPERATION CURVES

\section{2) Economic and Perceived Benefit Analysis of Two-stage} Optimization Model

In order to examine the economic and perceived benefits of the two-stage energy optimization, this paper compares the economic benefits of the two-phase optimization with three cases, and the results are shown in Table 4 . In case 1, consumers adopt traditional strategy. Case 2 reflects the first stage of the proposed optimization strategy. Case 3 reflects the complete optimization strategy. Case 4 reflects the first stage of the proposed optimization strategy ignoring consumption perceived benefits.
TABLE IV. COMPARISON OF FOUR ELECTRICITY PURCHASE STRATEGIES

\begin{tabular}{|c|c|c|}
\hline Strategy & System Running Cost & Perceived Benefits \\
\hline Case 1 & 21.53 & 11.42 \\
\hline Case 2 & 19.77 & 10.31 \\
\hline Case 3 & 15.96 & - \\
\hline Case 4 & 18.25 & 9.51 \\
\hline
\end{tabular}
formulations (8) and (11), Calculation of power satisfaction references formulations (5) and (7). As can be seen from Table 4 , under the traditional method, the system operating cost is 21.53. In the smart home optimization control model, the system running cost in the first stage is 19.77; the system running cost in the second stage is 15.96; and the system running cost in the second group is 18.25. Obviously, the economic costs of using the optimal scheduling algorithm are much lower than those of the two control groups. In addition, due to the impact of time load, users' satisfaction under the optimization strategy is slightly less than that of the traditional method, reducing by $9.1 \%$, but still superior to that of case 4 .

\section{CONCLUSION}

This article does the following work for energy management of demand-side smart home system:

1) The load control model and the energy storage control model are established to form a two-phase intelligent house optimization model.

2) The load control problem is solved by genetic algorithm, and the energy storage control problem is solved by the particle swarm optimization algorithm. The optimal solution of the first-stage optimal control takes part in the second-stage optimal control as the basic load and the minimum objective function constraint.

Throughout the optimization cycle, the state of charge changes gently. Considering that the energy storage system has the characteristics of buffered electric energy to stabilize the load fluctuations and uninterrupted power supply, it is beneficial to the energy distribution of the power grid. The economic benefits of the two-stage optimal control algorithm are much higher than the traditional method. Under the circumstance of energy optimization and dispatching, the smart home system encourages terminal consumers of power grid to take advantage of the controllability of flexible load and the storage energy.

\section{ACKNOWLEDGMENT}

The research is supported by the project in National Key Research and Development Program: (Grant No: 2017YFB 0903700): Prospective Study on the Seawater Pumping Storage Power Station Technology.

\section{REFERENCES}

[1] ZENG Ming, WANG Dongrong, CHEN Zhen, et al. Application of demand side response in electricity retail market[J].Power Demand Side Management, 2009, 11(1):13-16.

[2] WANG Yilan, TONG Yibin, HUANG Mei, et al. Research on Virtual Energy Storage Model of Air Conditioning Loads Based on Demand Response[J]. Power System Technology, 2017, 41(2):394-401. 
[3] YANG Yan, CHEN Haoyong, ZHANG Yao, et al. An Electricity Market Model With Distributed Generation and Interruptible Load Under Incomplete Information[J].Proceedings of the CSEE, 2011, 31(28):15-24.

[4] MA Lin, ZHANG Shirong. Optimal Operation Scheduling of Family GridConnected Photovoltaic Systems Under Time-of-Use and Ladder Tariffs[J]. Power System Technology, 2016(3): 819-825.

[5] Celik B, Roche R, Bouquain D, et al. Decentralized neighborhood energy management with coordinated smart home energy sharing[J]. IEEE Transactions on Smart Grid, PP(99):1-1.

[6] Pilloni V, Floris A, Meloni A, et al. Smart Home Energy Management Including Renewable Sources: A QoE-driven Approach[J]. IEEE Transactions on Smart Grid, 2016, PP(99):1-1.

[7] LUAN Wenpeng, WANG Guan, XU Daqing .Advanced Metering Infrastructure Solution Supporting Multiple Services and Business Integration[J]. IEEE Transactions on Smart Grid, 2014, 34(29):5088-5095.

[8] ZENEG Cheng.The load time elasticity identification and its application to orderly power utility[D]. Hunan University,2013.

[9] Qayyum F A, Naeem M, Khwaja A S, et al. Appliance scheduling optimization in smart home networks comprising of smart appliances and a photovoltaic panel[C]// Electrical Power and Energy Conference. IEEE, 2016:457-462.

[10] Cugnet M, Liaw B Y. Effect of discharge rate on charging a lead-acid battery simulated by mathematical model[J]. Journal of Power Sources, 2011, 196(7): 3414-3419.

[11] Dubarry M, Liaw B Y, Chen M S, et al. Identifying battery aging mechanisms in large format Li ion cells[J]. Journal of Power Sources, 2011, 196(7): 3420-3425.

[12] Broussely M, Biensan P, Bonhomme F, et al. Main aging mechanisms in Li ion batteries[J]. Journal of Power Sources, 2005, 146(1): 90-96.

[13] HuangB J, Hsu P C, Wu M S, et al. System dynamic model and charging control of lead-acid battery for stand-alone solar PV system[J]. Solar Energy, 2010, 84(5) : 822-8 